\title{
LANGUAGE AS A PARASITE TO EMOTIONAL SIGNALS
}

\author{
KAZUO OKANOYA \\ cokanoya@mail.ecc.u-tokyo.ac.jp \\ Department of Life Sciences, The University of Tokyo, Tokyo, Japan
}

The evolution of speech poses a dilemma when viewed from the signaling theory in evolutionary biology (Searcy \& Nowicki, 2005). The dilemma comes from dual nature of speech. First, the content of speech, linguistic expressions, is not honest. This is because the content is not always grounded with the reality and by compositional nature of words and sentences, expression is infinite. Furthermore, the cost of linguistic content is quite low (Lachmann et al, 2001). The speaker can easily transmit false information regarding with the cost/benefit of the hearer. Trust must be established between the speaker and hearer before taking the linguistic message but such process itself is costly (Dawkins \& Guilford, 1991). Second, the act of speech including bodily movement, facial expression, prosody, loudness, fluency, and vocabulary are mostly honest signals for health, intelligence, and genetic fitness. The creativity of language rests on the "ungrounded-ness" of linguistic content that is open to false information. If so, why did such a dishonest signal evolved at all?

I will lay out a hypothesis on this issue. Acoustical communication in terrestrial animals perhaps started as noises contingent with breathing and jaw or respiratory gestures associated with predation or feeding. These noises and bodily movements were gradually ritualized, forming the "fixed action patterns" of motor actions reflecting intentional or emotional states (Newman, 2012). Especially, vocalizations associated with respiratory action became indicators of emotional states such as fear or contentment, or that of intentions such as attack or copulation. Animal calls were thus established as an honest indicator of the internal state.

In certain species, stochastic combinations of such calls were used by young animals to induce parental behavior, perhaps because they reminded parents of immature articulation. This effect was then utilized by male animals to attract, or to suppress escape behavior of females. Extremes of such vocalizations are songs, used for mate attraction and/or territorial defense by many species of birds and some species of whales and primates (Fitch, 2006). 
Songs are an honest signal of vigor, since singing is costly in terms of nutrition, safety, and time. Songs came to be utilized also in non-sexual social contexts seen in, for example, gibbons (Clarke, Reichard, \& Zuberb hler, 2006).

Sequences of song syllables and behavioral contexts were gradually associated through a mutual segmentation process (Merker \& Okanoya, 2007; Okanoya \& Merker, 2007) and proto-words emerged. Arbitrary combinations of proto-words referred to non-existing entities and thus the dishonesty of speech started. However, speech was always associated with honest signals such as emotional expression in voice or facial. It is also suggestive that although fundamental frequency can be modified rather easily, changing formant frequencies are not as easy in human speech, securing honesty of speech output (Pisanski et al, 2014).

Thus, the receiver could mostly judge the honesty of the speech content. In this way, language content was able to evolve as parasitic to emotional expressions associated with the act of speech.

Furthermore, the dishonesty of linguistic expression produced creativity, and eventually, cumulative and transmittable culture. These byproducts provided strong survival value to humans as species competing with larger predator animals. In this way, linguistic contents and speech expression evolved in humans because of the mutual dependency between dishonesty and creativity.

\section{Acknowledgements}

Supported in part by This study was supported by MEXT Innovative Areas \#4903, JP17H06380 to K.O.

\section{References}

Clarke, E., Reichard, U. H., \& Zuberbühler, K. (2006). The syntax and meaning of wild gibbon songs. PLOS ONE, $1(1)$, e73.

Dawkins, M. S., \& Guilford, T. (1991). The corruption of honest signalling. Animal Behaviour, 41(5), 865-873.

Fitch, W. (2006). The biology and evolution of music: A comparative perspective.Cognition, 100 (1), 173-215.

Lachmann, M., Szamado, S., \& Bergstrom, C. T. (2001). Cost and conflict in animal signals and human language. Proceedings of the National Academy of Sciences, 98(23), 13189-13194.

Merker, B., \& Okanoya, K. (2007). The natural history of human language: Bridging the gaps without magic. Emergence of communication and language, 403-420.

Newman, J. D. (2012). The physiological control of mammalian vocalization: Springer Science \& Business Media.

Okanoya, K., \& Merker, B. (2007). Neural substrates for string-context mutual segmentation: a path to human language. Emergence of communication and 
language, 421-434.

Pisanski, K., Fraccaro, P. J., Tigue, C. C., O'Connor, J. J., Röder, S., Andrews, P. W., ... \& Feinberg, D. R. (2014). Vocal indicators of body size in men and women: a meta-analysis. Animal Behaviour, 95, 89-99.

Searcy, W. A., \& Nowicki, S. (2005). The evolution of animal communication: reliability and deception in signaling systems: Princeton University Press. 